Revue musicale OICRM

\title{
Le(s) numéro(s) à instruments du film musical hollywoodien
}

\section{Marguerite Chabrol}

Volume 5, numéro 1, 2018

Ce que le cinéma nous apprend de la musique

URI : https://id.erudit.org/iderudit/1044441ar

DOI : https://doi.org/10.7202/1044441ar

Aller au sommaire du numéro

Éditeur(s)

Observatoire interdisciplinaire de création et recherche en musique (OICRM)

ISSN

2368-7061 (numérique)

Découvrir la revue

Citer cet article

Chabrol, M. (2018). Le(s) numéro(s) à instruments du film musical hollywoodien. Revue musicale OICRM, 5(1), 7-32.

https://doi.org/10.7202/1044441ar

\section{Résumé de l'article}

Parmi ses multiples thèmes de numéros, le cinéma musical hollywoodien a attaché une importance particulière à ceux représentant des instruments et instrumentistes dans un acte de performance, au point d'en faire une attraction type, que cet article examine sous un double angle historique et esthétique. Ces numéros proviennent d'abord d'un positionnement de Hollywood par rapport à deux médias concurrents : le théâtre musical de Broadway et la radio. Ils permettent à l'industrie du cinéma de proposer un spectacle propre tout en bénéficiant de la circulation des musiciens. Ces numéros se construisent ensuite autour d'une poétique précise, relevant d'emprunts à des traditions iconographiques et d'un travail sur la musicalité visuelle. On peut ainsi mettre au jour des formes communes au-delà des styles de musique concernés : le jazz, le swing, les musiques latines ou classiques. Les numéros à instruments se présentent ainsi comme un des lieux essentiels du syncrétisme culturel pratiqué par le cinéma classique américain. 


\title{
Le(s) numéro(s) à instruments du film musical hollywoodien
}

\author{
Marguerite Chabrol
}

\begin{abstract}
Résumé
Parmi ses multiples thèmes de numéros, le cinéma musical hollywoodien a attaché une importance particulière à ceux représentant des instruments et instrumentistes dans un acte de performance, au point d'en faire une attraction type, que cet article examine sous un double angle historique et esthétique. Ces numéros proviennent d'abord d'un positionnement de Hollywood par rapport à deux médias concurrents : le théâtre musical de Broadway et la radio. Ils permettent à l'industrie du cinéma de proposer un spectacle propre tout en bénéficiant de la circulation des musiciens. Ces numéros se construisent ensuite autour d'une poétique précise, relevant d'emprunts à des traditions iconographiques et d'un travail sur la musicalité visuelle. On peut ainsi mettre au jour des formes communes au-delà des styles de musique concernés : le jazz, le swing, les musiques latines ou classiques. Les numéros à instruments se présentent ainsi comme un des lieux essentiels du syncrétisme culturel pratiqué par le cinéma classique américain.
\end{abstract}

Mots clés: Hollywood ; numéros musicaux ; instrumentistes ; intermédialité ; attractions.

\begin{abstract}
The Hollywood film musical featured, among all kinds of production numbers, some presenting instruments and instrumentalists shown while performing. This became a typical attraction, which this article tackles in a historical and aesthetical perspective. These numbers can be related to Hollywood's position between two other media: the Broadway stage and the radio. The film industry thereby offers an original spectacle while benefiting from the musicians' circulation across media spaces. Aesthetically, the numbers are directed and edited following recurring principles, on the one side imitations of other iconographic traditions and on the other side the design of a of visual musicality. Common forms can be identified beyond the musical styles involved in those numbers (jazz, swing, latin or classical music). Numbers with instruments thus appear as an important part of the Hollywood cultural syncretism.
\end{abstract}

Keywords: Hollywood; musical numbers; instrumentalists; intermediality; attractions. 
Parmi les fondements qu'il définit pour le genre de la comédie musicale, Alain Masson envisage, avec le corps ou l'érotisme, les "types de numéros » (Masson 1981, p. 131), pour lesquels il adopte une présentation par effectif, c'est-à-dire l'interprétation en solo, duo, etc. En effet, toute tentative de typologie des numéros "selon leur style ou leur contenu » (ibid.) lui apparaît comme nécessairement délicate compte tenu de l'ampleur du corpus. De son côté, Rick Altman caractérise la " sémantique " du sous-genre de la comédie musicale "spectacle » comme issue du théâtre de variétés américain (Altman 1987, p. 201). Il insiste en particulier sur le fait que le film musical de ce sous-genre emprunte en particulier au vaudeville trois aspects majeurs : interprètes, formats et thématiques. Si les deux auteurs n'en font pas leur préoccupation centrale, ils pointent chacun à leur manière la difficulté d'une typologie des contenus thématiques et stylistiques des numéros musicaux. Si l'exercice taxonomique peut sembler vain dans sa globalité, repérer des modèles récurrents à cette échelle et retracer les filiations entre les médias permet néanmoins d'aborder le genre sous un angle nouveau.

Ainsi, une grande partie des formes travaillées par Busby Berkeley au cinéma ont été identifiées par Martin Rubin comme des archétypes issus de pratiques scéniques, par exemple les numéros avec des éclairages au néon ("Radium numbers »), le recours au gigantisme, les métamorphoses, les numéros aquatiques... (Rubin 1993, p. 61). Il ne s'agit pas simplement de motifs ponctuels, mais bien de types récurrents d'attractions. Parmi celles-ci, Rubin caractérise notamment les «numéros dans lesquels les instruments de musique figurent au premier plan ${ }^{1}$ ( ibid.) parl'autonomie que semblent prendre les objets et l'effacement de l'instrumentiste, absent ou parfois transformé en ombre - par exemple les célèbres pianos de "The Words Are in My Heart » (extrait vidéo 1) dans Gold Diggers of 1935 (Busby Berkeley, 1935) ou " Fascinatin' Rhythm », le finale dirigé par Berkeley pour Lady Be Good (Norman Z. McLeod, 1941).

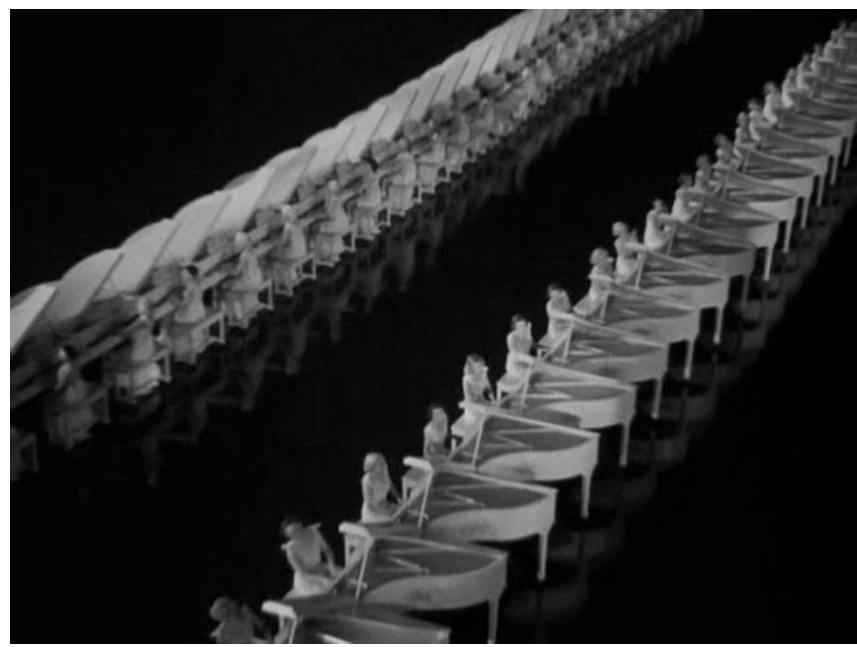

Extrait vidéo 1 : Busby Berkeley, Gold Diggers of 1935 (1935), "The Words Are in My Heart» (C Warner Bros.

1 «Numbers in which musical instruments figure prominently» (traduction personnelle). 
Rubin a retracé les origines scéniques de ces attractions, citant notamment des revues comportant des instruments humains (grâce à l'usage de déguisements en pianos ou violons) et des numéros de grand ensemble, comme dans les Ziegfeld Follies de 1927, dont le finale du premier acte réunit un jazz band de girls, une douzaine de pianos dorés et une fanfare militaire en parade (Rubin 1993, p. 62).

Au-delà du seul style de Berkeley, et bien que les études sur la comédie musicale les mentionnent assez peu comme des moments majeurs, de nombreux numéros de films musicaux (ou des segments importants de numéros) reposent simplement sur le fait de montrer les musiciens en action avec leurs instruments, sans nécessairement recourir à une autre dominante spectaculaire : il n'y a alors d'autre enjeu que les musiciens ni en matière de performance (jouer la comédie, danser) ni sur le plan narratif. Dans une partie de ces numéros de pure musique, liés autant à l'émergence des big bands pendant l'ère du swing qu'à des traditions théâtrales, des musiciens ou ensembles célèbres interviennent, souvent dans leur propre rôle ${ }^{2}$.

Le but de cet article est de définir historiquement et esthétiquement cette forme du "numéro à instruments ", en tant qu'archétype spectaculaire du film musical hollywoodien, c'est-à-dire un contenu type (la représentation de musiciens en action) et ses principales déclinaisons formelles. Je soulignerai les chaînons manquants entre les objets géants à la Ziegfeld ou Berkeley et la représentation parfois plus réaliste de big bands qui semble a priori relever d'une esthétique différente, sur le plan sonore comme visuel. À travers cette définition, j'envisagerai la vogue des numéros impliquant les big bands (de la deuxième moitié des années 1930 à la fin de la Seconde Guerre mondiale) dans le réseau de relations intermédiales dans lequel le cinéma en particulier musical - est pris dès ses débuts. En effet, l'air du temps et le succès des danses de couples (social dancing) au début des années 1940 ne suffisent pas à expliquer l'intérêt que Hollywood a alors prêté à la représentation des musiciens : les big bands occupent un espace " disponible», déjà existant dans différentes traditions spectaculaires, auquel ils donnent une coloration de modernité. Mais ils ne sont pas les seuls et un dialogue s'installe aussi dans les numéros à instruments entre différents styles issus des musiques noires, latines et classiques.

Le corpus entier serait évidemment immense. Pour proposer ici quelques hypothèses, destinées à des approfondissements ultérieurs, je m'appuie sur un échantillon constitué dans le cadre d'un projet de recherche collaboratif en humanités numériques ${ }^{3}$ : dans ce corpus de 154 films musicaux contenant un peu plus de 1900 numéros (il s'agit d'environ $10 \%$ de la production hollywoodienne entre 1929 et 1972), 44 films comportent

2 Sur les films de big bands, voir Chapman 2006.

3 Ces données sont issues du projet de recherche Musical $\mathrm{MC}^{2}$ (Labex Arts- $\mathrm{H} 2 \mathrm{H}$, Paris, http://labexarts-h2h.univ-paris8.fr/mc2/, 2015-2017), qui développe une plateforme numérique indexant les numéros musicaux des films à partir de certaines de leurs caractéristiques formelles. La plateforme, pour l'instant réservée au groupe de chercheurs internationaux associés au projet, sera rendue publique à l'adresse http://mc2.labex-arts-h2h.univ-paris8.fr/. Les données utilisées pour cet article sont amenées à évoluer et sont encore mises à jour. Cette étude s'appuie sur l'état de la base de données au $1^{\text {er }}$ septembre 2017 et les chiffres visent seulement ici à donner des ordres de grandeur sans prétendre être absolus. 
116 numéros à instruments entre 1929 et 1954. La moitié consiste en des versions purement instrumentales (éventuellement avec une section dansée mais sans chanteur ou chanteuse). L'autre moitié, contenant au moins une section chantée, comporte une forte proportion de numéros exotiques (essentiellement latins). J'ajouterai aux données chiffrées liées à cet échantillon des commentaires sur quelques séquences d'autres films montrant les musiciens vedettes de la période comme Benny Goodman, Xavier Cugat ou le chef Leopold Stokowski.

\section{LOGIQUES INTERMÉDIALES}

Des modalités variées d'intégration aux films

L'ère du swing, entre la deuxième moitié des années 1930 et le début des années 1940 est comme on peut s'y attendre marquée par un pic de numéros à instruments (figure 1). L'année 1938 est assez riche avec dans l'échantillon deux films de la Twentieth Century-Fox : Alexander's Ragtime Band (Henry King) et Happy Landing (Roy Del Ruth). Mais, si le lien est indéniable, il faut aussi le relativiser légèrement en soulignant que les numéros à instruments sont présents en proportion régulière de la production globale dès 1929/1930.

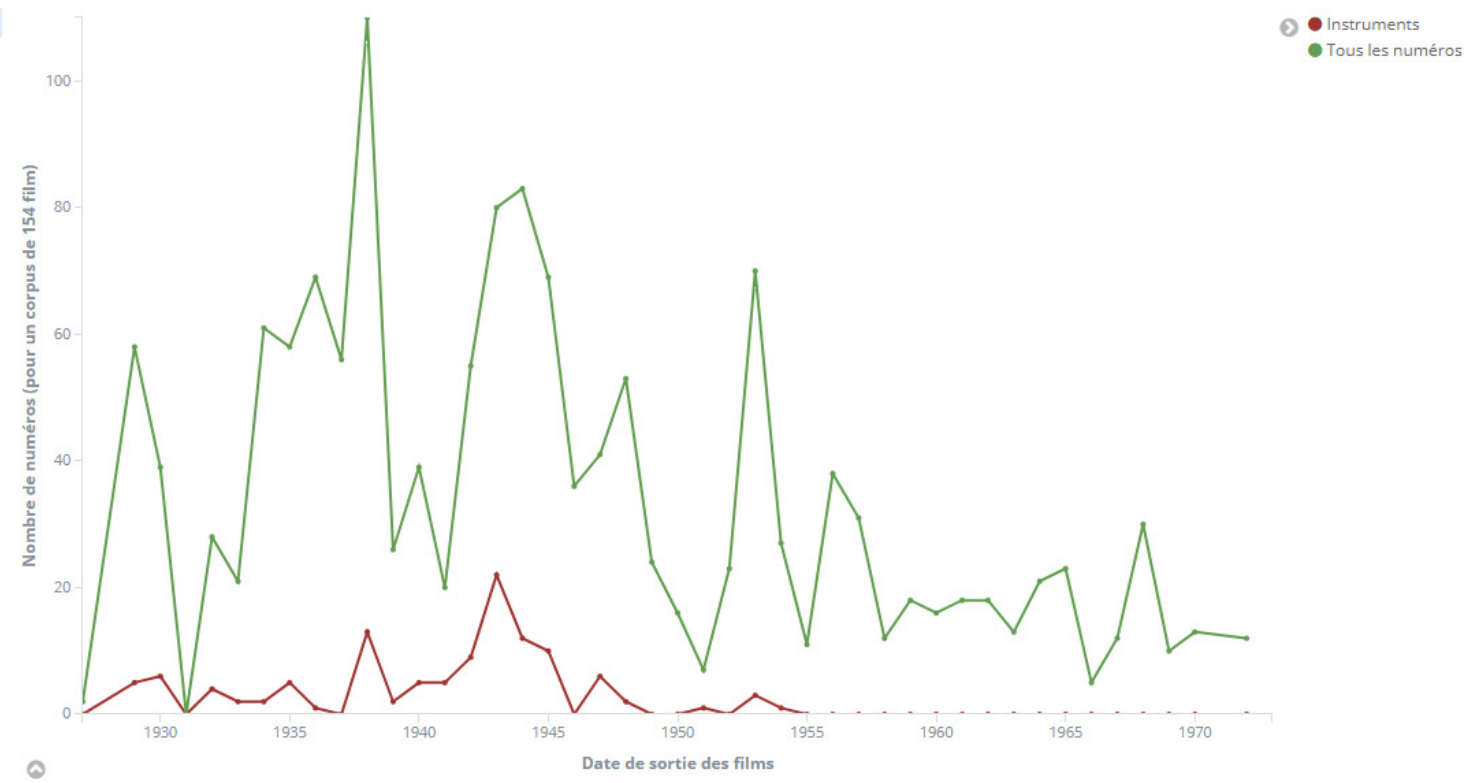

Figure 1 : Répartition chronologique des numéros à instruments dans le corpus étudié (visualisation réalisée avec Kibana/Elastic Search).

Il y a en effet une continuité entre les courts ou moyens métrages musicaux qui se développent progressivement dans les années $1920^{4}$, les longs métrages de revue des débuts du parlant qui intègrent des numéros centrés sur des instrumentistes dans 
différentes formations (comme King of Jazz (John Murray Anderson, 1930)) et les numéros de big bands. Tous contiennent des séquences musicales courtes analogues, indépendamment du format cinématographique qui les intègre.

Dans le cas des longs métrages, certains numéros à instruments constituent des occurrences uniques dans le film, en contribuant à en diversifier les attractions. D'autres sous-genres du musical se structurent en revanche progressivement autour de plusieurs numéros à instruments : il peut s'agir de films dont la musique relève presque exclusivement d'un seul groupe (par exemple les musicals de la Fox avec l'orchestre de Glenn Miller, Sun Valley Serenade (H. Bruce Humberstone, 1941) et Orchestra Wives (Archie Mayo, 1942)) et qui déclinent alors toutes les modalités de la représentation des instrumentistes (entre l'orchestre comme toile de fond à une action dansée et un véritable numéro à instruments, en passant par des scènes de répétitions, parfois interrompues). D'autres films ont recours au schéma de la confrontation en se présentant comme un concours de style musicaux, par exemple Bathing Beauty (George Sidney, 1944) qui oppose le groupe latin de Xavier Cugat à l'orchestre du trompettiste Harry James, oscillant entre virtuosité concertante classique et big band. Enfin, certains films relatent l'ascension d'un musicien et d'un groupe fictifs et leur trajectoire depuis les petites salles jusqu'à la gloire des grands auditoriums (comme Alexander's Ragtime Band, ou Strike Up the Band [Busby Berkeley, 1940]), avant l'émergence de biopics de musiciens réels ${ }^{5}$.

\section{Entre théâtre et radio}

Cette façon d'intégrer les numéros à instruments dans des sous-genres propres au musical hollywoodien ne doit pas dissimuler l'importance des logiques intermédiales. En effet, l'industrie du cinéma ne se contente pas d'inventer des récits pour justifier la présence de tel ou tel musicien. Elle joue en fait dans ces numéros sa double relation avec le théâtre et la radio.

D'une part la tradition scénique demeure, même si les numéros hollywoodiens ne s'inscrivent pas tous dans l'esthétique de l'extravaganza évoquée à propos de Berkeley. Le lien entre cinéma et scène musicale, n'implique pas seulement le théâtre de Broadway, mais aussi d'autres pratiques de la musique live, comme les variétés (de nombreux "specialty acts » sont des numéros d'instrumentistes sérieux ou comiques), et la musique intégrée à des programmes mixtes, combinant film de long métrage, courts métrages (cartoons, actualités, bandes-annonces...) et musique sur le plateau. L'une des plus célèbres salles spécialisées dans ces programmes est le Paramount Theatre de New York, construit à Times Square en 1926 pendant l'ère du jazz et contenant plus de 3000 sièges. Ce théâtre, connu notamment pour ses solos d'orgue donnés sur l'instrument local, avait surtout pour pratique d'accueillir dans la plupart des séances un groupe jouant live avant ou après le film. Les musiciens présents dans les films musicaux sont aussi en partie ceux que l'on peut entendre et voir en chair 
et en os dans cette salle en particulier. Ils y tenaient des sessions de 40 minutes à une heure, un peu moins longues que le film de fiction (d'environ 1h30), mais largement aussi intenses $d u$ fait de la dimension vivante $d u$ concert $^{6}$. La publicité met d'ailleurs ces deux composantes du spectacle quasiment à égalité (figure 2). Des années 1930 aux années 1950, le Paramount Theatre propose constamment ce type de programmation mixte, associant parfois, mais pas toujours, le concert à un film musical. Le groupe joue parfois des reprises ou un medley des morceaux du film. Il se produit plusieurs fois par jour (environ 5), dans la majeure partie des séances. En 1940, la durée des sessions musicales tend à s'allonger : par exemple Tommy Dorsey joue plus d'une heure en janvier 1941, parallèlement à la projection de Love Thy Neighbour (Mark Sandrich, 1940).

Figure 2. Publicité pour une séance du Paramount Theatre associant le film The Big Broadcast of 1938 (Mitchell Leisen) avec un concert du groupe de Cab Calloway.

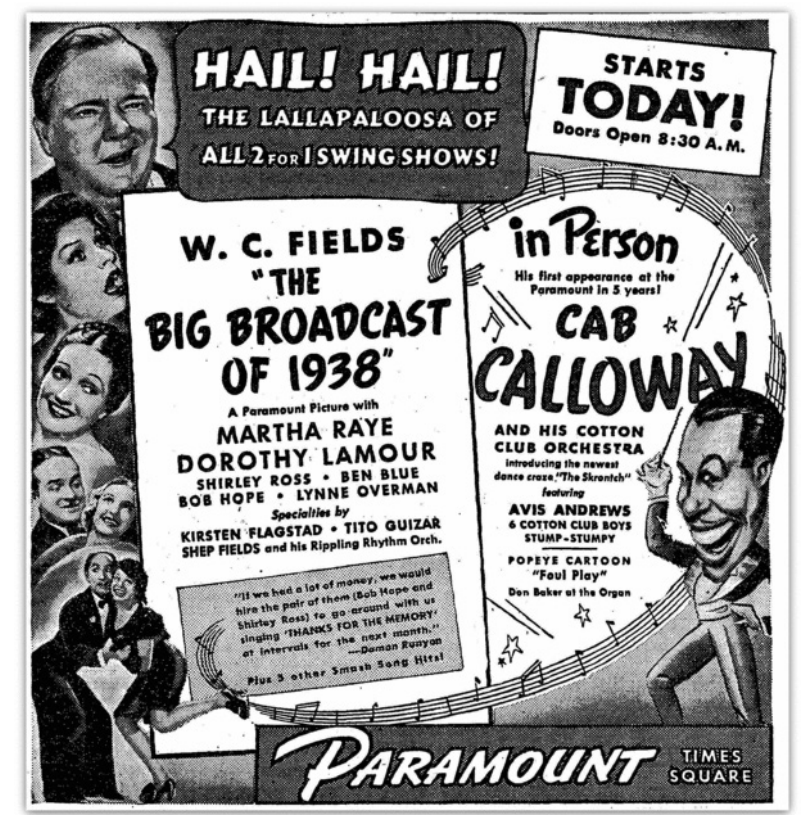

Ces programmations du Paramount Theatre cessent progressivement dans les années 1950 : les dernières dates de concerts mentionnées sont en 1954/1955 et c'est aussi le moment où les numéros à instruments disparaissent des films ${ }^{7}$. En 1954, A Star Is Born passe au Paramount Theatre sans programme musical conjoint, puisque le film à lui seul occupe l'intégralité d'une séance. Mais il est significatif que ses deux premiers numéros évoquent ce dialogue entre spectacle vivant et numéros à instruments cinématographiques : le premier, ("Gotta Have Me Go with You ») (extrait vidéo 2), sur scène, est filmé de façon relativement frontale pour souligner le dispositif théâtral. Le big band est à l'arrière-plan, en bloc, sans que la mise en scène lui accorde des plans rapprochés et en fasse une attraction à part entière. Le deuxième,

6 La durée des sessions est indiquée dans les archives du régisseur la salle (Paramount Theatre Stage Manager's Records) conservées à la New York Public Library, Collections des arts du spectacle au Lincoln Center. Toutes les informations données ici proviennent de ce fonds.

7 Même si plus généralement le film musical décline à cette époque, la figure 1 montre que sur l'échantillon de numéros musicaux analysés après 1954, il n'y a plus de numéros à instruments. 
"The Man That Got Away » (extrait vidéo 3), est un véritable numéro à instruments où l'image est structurée par les lignes des cuivres ou des bois plutôt qu'elle ne renvoie à la scène. Marquant la fin d'une époque, ce film illustre à quel point les numéros à instruments sont en dialogue constant avec le spectacle vivant, par rapport auquel ils recherchent une forme de démarcation.

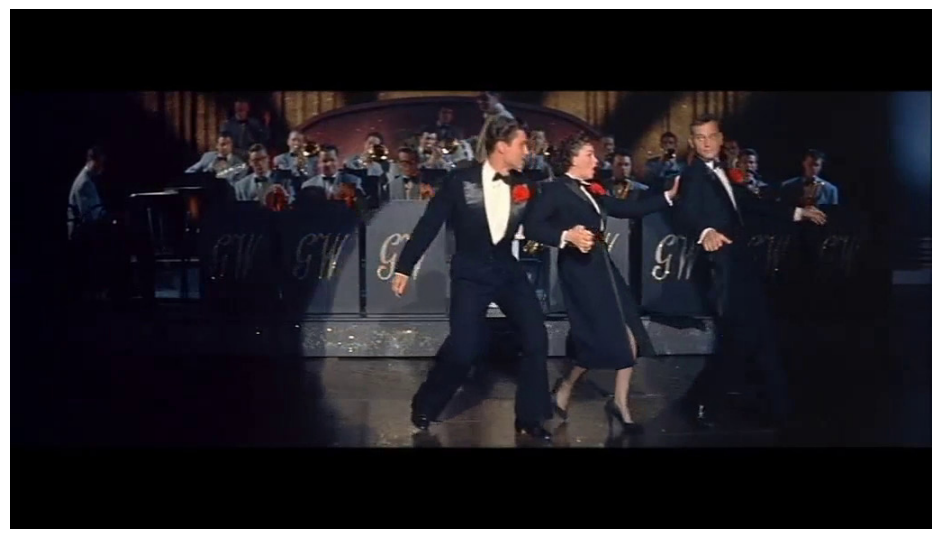

Extrait vidéo 2 : George Cukor, A Star Is Born (1954), Judy Garland dans "Gotta Have Me Go with You " (C) Warner Bros.

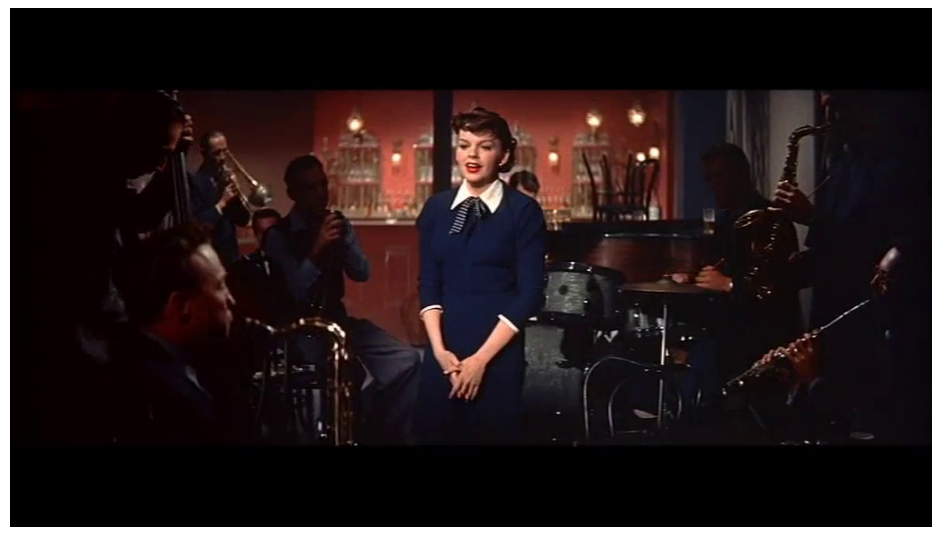

Extrait vidéo 3 : George Cukor, A Star Is Born (1954), Judy Garland dans "The Man That Got Away»

(C) Warner Bros.

D'autre part, l'évolution des numéros à instruments dans les films fait directement écho à l'explosion de la radio comme média de masse au cours des années 1930, où les émissions de variétés prolifèrent. Beaucoup de films de cette décennie, en particulier la série des Big Broadcast de Paramount, s'appuient directement sur le modèle des variétés radiophoniques et il est très fréquent de voir les micros de radiodiffusion mis en évidence dans les cadrages des numéros à instruments, même quand ils ne servent pas à justifier des séquences de montage, par exemple entre le studio et des spectateurs à l'extérieur.

La radio aussi est un média du direct. Par rapport aux deux médias voisins diffusant le même type de musique, le cinéma est ainsi confronté à la perte de l'aura des musiciens. Pourtant, tous les numéros cinématographiques montrant les musiciens n'ont pas recours à des compensations spectaculaires, notamment en prenant le parti de l'artifice et des effets techniques qui marqueraient une forme de surenchère 
par rapport aux caractéristiques du concert live. On trouve beaucoup de séquences musicales qui, loin de montrer des instruments factices, automatisés, pris dans des effets spéciaux, misent au contraire sur l'idée du "vrai ", et insistent sur la simple performance des instrumentistes et la coordination des gestes et des objets. Hollywood a véritablement fait confiance à cette forme dans la mesure où, par comparaison avec chacun des médias rivaux, le cinéma donne un meilleur accès aux musiciens : par rapport à une scène comme celle du Paramount Theatre, le cinéma touche un plus vaste public dans des endroits plus diversifiés, de même que les cadrages rapprochés révèlent les instrumentistes avec une proximité inédite ; et par rapport à la radio, quant à elle plus répandue, le cinéma possède évidemment la faculté de montrer ${ }^{8}$. Dans les deux cas Hollywood mise en fait sur sa capacité à donner une lisibilité exceptionnelle à la musique.

\section{Une démarcation par rapport à Broadway}

La force de l'attraction que représentent les musiciens semble même les rendre plus intéressants pour l'industrie du cinéma que le théâtre musical de Broadway. Les films que j'ai pu examiner suggèrent que les numéros à instruments se sont notamment inscrits dans une stratégie de démarcation du cinéma par rapport au genre musical tel qu'il se développait parallèlement sur les scènes new-yorkaises. Si l'on regarde les sources de ces numéros, très peu proviennent en réalité du théâtre par comparaison avec l'ensemble du genre musical, qu'il s'agisse de numéros tirés du spectacle d'origine quand le film est une adaptation, ou d'une chanson empruntée à un spectacle de théâtre, indépendamment de son adaptation cinématographique (figure 3).

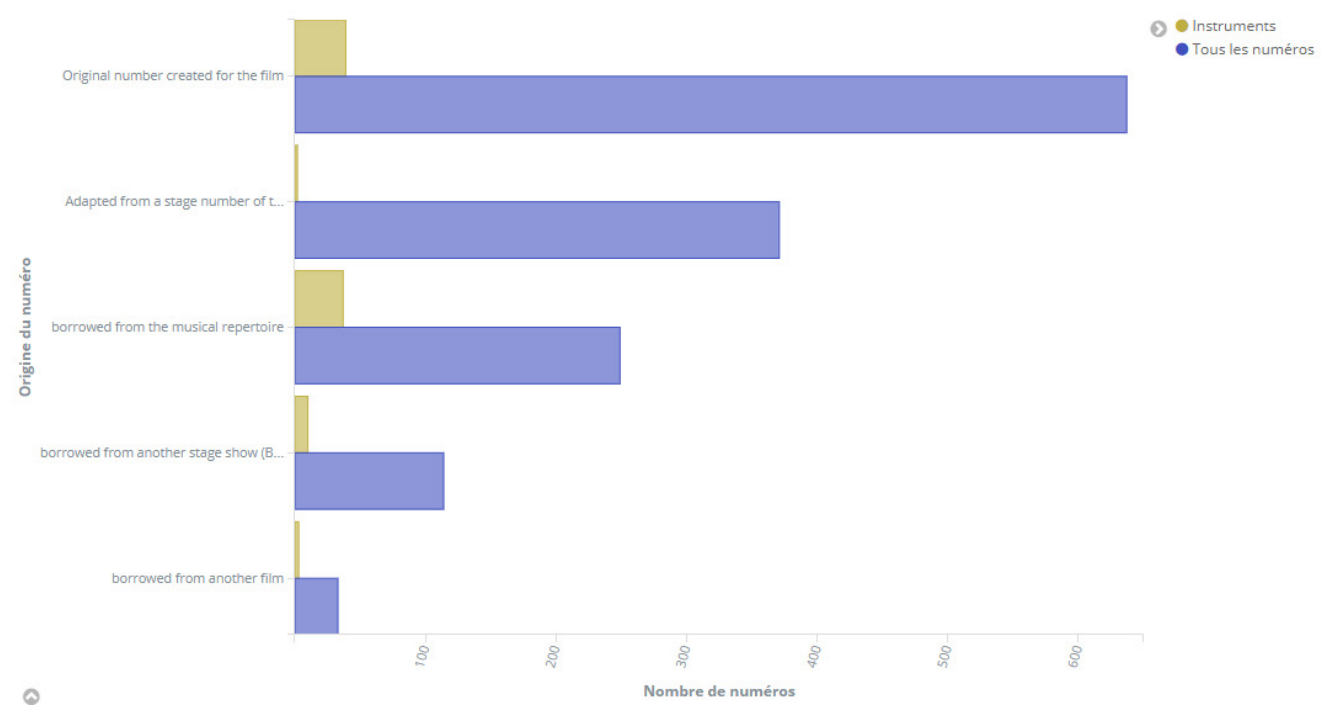

Figure 3 : Sources des numéros à instruments par rapport à l'ensemble des numéros musicaux hollywoodiens étudiés (visualisation réalisée avec Kibana/Elastic Search).

8 Voir Altman 1999, qui traite de la période précédente, mais expose les enjeux théoriques de l'affirmation de supériorité d'un média par rapport aux autres. 
En fait, Hollywood met en concurrence Broadway et la radio dans ces numéros, en y recourant en particulier pour 1'adaptation de certains spectacles. En effet, on sait que les adaptations des musicals théâtraux sont assez libres avant la Seconde Guerre mondiale et que beaucoup de spectacles sont alors sensiblement transformés lorsqu'ils passent à l'écran. Mais on souligne moins que cette transformation s'est notamment faite par la "greffe " de numéros à instruments sur la trame d'un spectacle scénique : Best Foot Forward (Busby Berkeley, 1943), tiré d'un spectacle chorégraphié par Gene Kelly qui a tenu 326 dates à Broadway en 1941, est surtout dans sa version filmique centré sur la nouveauté : la virtuosité de Harry James et son orchestre. Du Barry Was a Lady (Roy Del Ruth, 1943) conserve la trame de la version scénique de 1939, mais laisse de côté l'essentiel de la partition de Cole Porter - pourtant très apprécié à Hollywood. Le film mise en particulier sur deux grands numéros centrés exclusivement sur l'orchestre de Tommy Dorsey, dont le deuxième ("Katie Went to Haiti ») repose sur un anachronisme consistant à faire jouer le big band en costumes français du XVIII siècle (extrait vidéo 4). Une "fausse » adaptation comme Strike Up the Band, qui n'a gardé de l'ancien musical que la chanson-titre, mise aussi entièrement sur le concept du big band en mettant en scène la fascination du groupe fictif de Mickey Rooney pour l'orchestre de Paul Whiteman qui apparaît sous son nom propre.

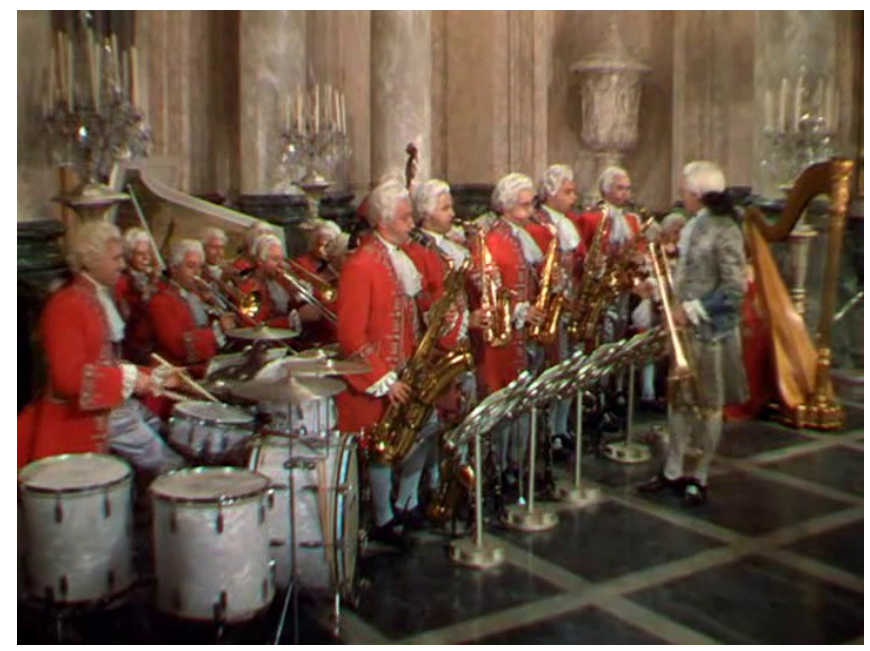

Extrait vidéo 4 : Roy Del Ruth, Du Barry Was a Lady (1943),

"Katie Went to Haiti " par Tommy Dorsey et son orchestre (C) MGM.

Il faudrait enquêter davantage d'un point de vue historique, mais ce principe de la " greffe » musicale semble avoir opéré avant l'ère du swing : dans la version filmique de The Merry Widow (Ernst Lubitsch, 1934), l'ajout de la prestation d'un groupe gitan vient prolonger au début la chanson "Vilia "; dans The Cocoanuts (Robert Florey et Joseph Santley, 1929), les versions instrumentales de «When My Dreams Come True " (avec Harpo à la clarinette puis Chico au piano) - une chanson écrite par Irving Berlin pour le film - apparaissent comme un contrepoint comique à la nécessité très hollywoodienne d'ajouter au spectacle d'origine une grande chanson romantique. En outre, le phénomène touche des répertoires musicaux variées, et notamment les musiques noires. Deux chansons «à instruments » sont ainsi ajoutées lors de l'adaptation cinématographique de Cabin in the Sky (Vincente Minnelli, 1943) : "Going Up », où 
Duke Ellington et son groupe plongent la foule en transe (extrait vidéo 5); et "Shine » avec John Sublett, dont la première partie montre les instrumentistes qui l'accompagnent. En matière d'attractions, elles remplacent les numéros dansés de la troupe de Katherine Dunham à Broadway.

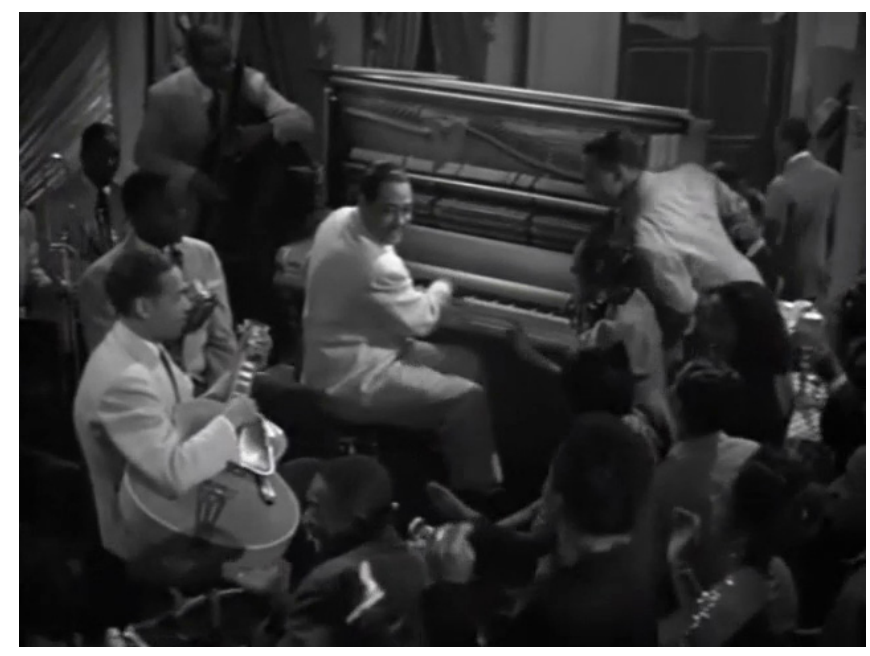

Extrait vidéo 5 : Vincente Minnelli, Cabin in the Sky (1943), "Going Up" par Duke Ellington et son orchestre (C) MGM.

En plus des « greffes » permettant des substitutions d'attractions, la forme du numéro à instruments sert parfois à transformer radicalement une chanson pré-existante dans la version scénique. "Too Darn Hot » dans Kiss Me, Kate (George Sidney, 1953) est au cinéma la combinaison d'un numéro de simili strip-tease d'Ann Miller doublé d'un numéro à instruments que la 3D stéréoscopique met en valeur en leur donnant notamment l'aspect d'objets géants à la Berkeley : les bongos apparaissent ainsi en très grande taille au premier plan, donnant l'impression de "sortir » de l'écran (figure 4). Sur scène, il s'agissait d'un intermède lui aussi à connotation érotique, mais conçu différemment puisque la chanson exprimait le moment de détente des employés noirs du théâtre, en coulisses de la représentation principale. Dans Du Barry Was a Lady, déjà évoqué, l'une des rares chansons conservées dans le film, "Katie Went to Haiti ", était sur scène une chanson risquée et gouailleuse d'Ethel Merman, alors que le film s'appuie plutôt sur le fait que le morceau a déjà été repris par Ray Noble et son orchestre. Ces deux exemples suggèrent que certaines chansons, en particulier celles aux paroles trop sulfureuses pour Hollywood, peuvent trouver une nouvelle énergie dans la forme du numéro à instruments. Parallèlement cette forme apparaît presque comme une « désintégration » volontaire du genre musical, qui résiste en fait assez longtemps à l'intégration narrative des numéros. 


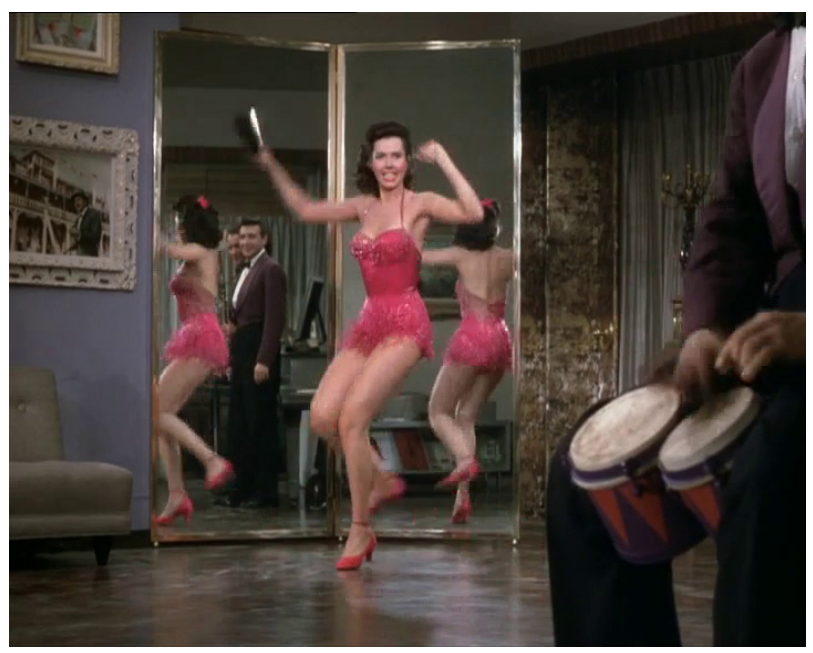

Figure 4: Ann Miller et les musiciens dans le numéro "Too Darn Hot». Photogramme de Kiss Me, Kate (George Sidney, 1953).

\section{RYTHMES ET ICONOGRAPHIE}

D'un point de vue esthétique, les façons de filmer les numéros à instruments sont variées et le dialogue avec le théâtre et la radio n'implique pas nécessairement un déferlement d'effets. Certains numéros se présentent comme assez simples, avec peu de montage, et conservent presque le style des captations montrées parallèlement dans les actualités filmées. Mais en général, les numéros les plus développés et ceux impliquant les musiciens les plus célèbres ont une forme très élaborée, à défaut d'être systématiquement ostentatoire.

Les numéros de virtuoses jouant de manière incongrue (de la catégorie du numéro de "specialty») reposent en général sur un plan fixe (ou un nombre limité de plans), insistant sur l'effet de captation et rappelant la tradition culturelle du vaudeville : c'est par exemple le registre fréquent des Marx Brothers pour leurs solos instrumentaux, ou du Professor Lamberti et son xylophone dans Tonight and Every Night (Victor Saville, 1945). D'autres scènes sont parfois filmées avec un découpage assez neutre minimisant les effets visuels pour insister sur la relation entre la foule et les musiciens et ainsi créer un sentiment de communauté, en particulier dans les numéros à visée patriotique, par exemple ceux du film Stage Door Canteen (Frank Borzage, 1943). Pour le numéro de Benny Goodman, «Bugle Call Rag (extrait vidéo 6), le découpage "suit " les différents solistes, mais avec des cadrages très neutres visant à donner le sentiment d'une saisie sur le vif.

Extrait vidéo 6 : Frank Borzage,

Stage Door Canteen (1943),

"Bugle Call Rag " par Benny Goodman

(C) Sol Lesser Productions.

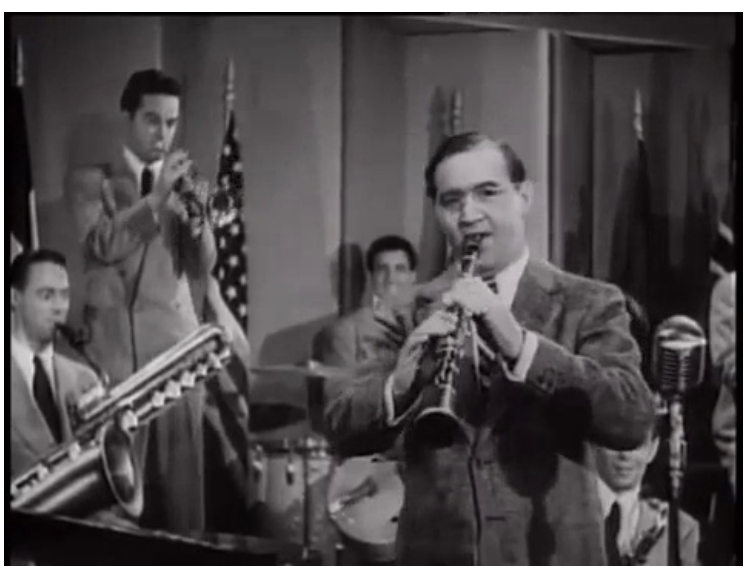


Mais la plupart du temps, ces numéros font l'objet d'une réalisation très structurée, située en fait au carrefour de trois principes : la tradition scénique des instruments-objets ; le style des photographies des groupes de jazz, en particulier noirs, du début du siècle à la Harlem Renaissance ; et une recherche visuelle liée à la pratique du montage cinématographique. Si les trois n'interviennent pas dans chaque numéro, les plus complexes peuvent les combiner.

\section{Les instruments objets}

Si tous les réalisateurs n'ont pas le style expressif de Berkeley, une partie a recours ponctuellement aux instrument gigantesques ou « automatisés ». Comme « Too Darn Hot ", d'autres numéros sont le lieu d'un déploiement d'effets spéciaux qui permettent d'obtenir des instruments géants d'une façon inédite par rapport au théâtre, tout en lui empruntant ses traditions. Le numéro " Rhapsody in Blue » dans King of Jazz (extrait vidéo 7) met d'emblée en scène un piano colossal et propose, dans sa première partie, une série de variations sur les différents rapports de taille entre musiciens et instruments. La même séquence superpose ainsi des gestes « vrais » (par exemple ceux des musiciens de l'orchestre) et des gestes absurdes mais vraisemblables (ceux des cinq petits pianistes qui se partagent le clavier du grand piano - lui-même présentant un nombre de touches irréaliste). Cette séquence mélange entièrement le vrai et le faux du geste instrumental et mise précisément sur ces décalages pour obtenir ses effets spectaculaires. Des principes décrits par Rubin (1993, p. 61), on retrouve aussi dans les films musicaux hollywoodiens l'autonomie de l'instrument et la désincarnation des musiciens : par des jeux de lumière cachant leurs visages, par des plans rapprochés isolant les instruments et les mains, et par des traitements des instruments en "série " pour les démultiplier, tous types d'effets auxquels a par exemple recours le numéro « Hora Staccato » de Bathing Beauty (extrait vidéo 8).

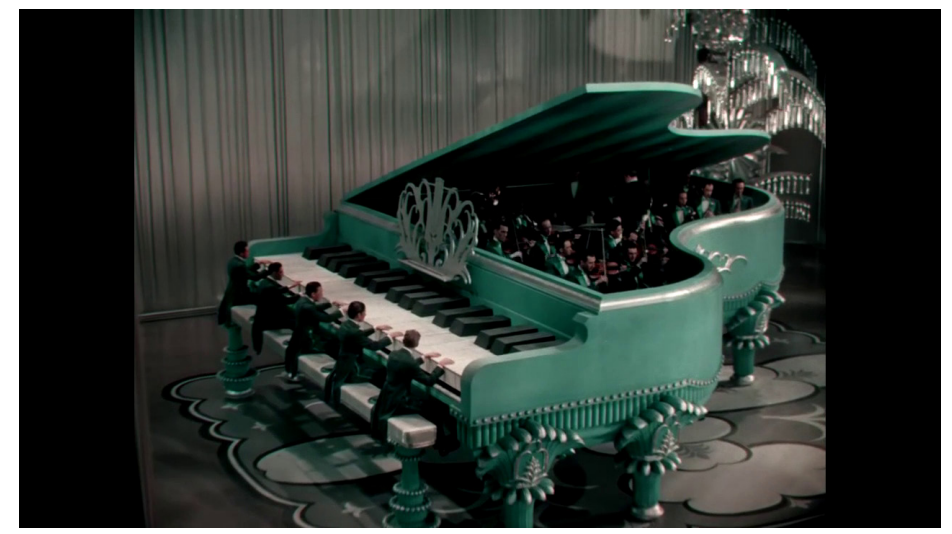

Extrait vidéo 7 : John Murray Anderson, King of Jazz (1930), «Rhapsody in Blue», (C) Universal. 
Extrait vidéo 8: George Sidney,

Bathing Beauty (1944), "Hora Staccato " par Harry James (C) MGM.

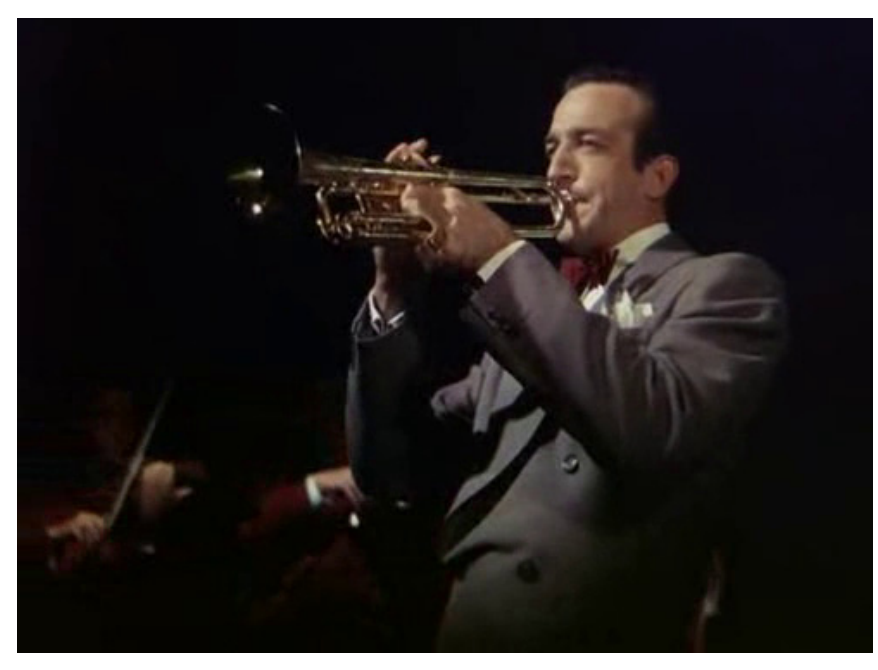

L'inspiration des photos des premiers groupes de jazz

Les choix de composition de l'image ne s'expliquent pas seulement par le fait de donner une forme cinématographique à une figure théâtrale. Ils déclinent aussi des principes issus des traditions photographiques associées à la représentation des groupes de jazz, assez travaillée sur le plan visuel ${ }^{9}$. Ces photos font souvent poser les groupes en position dynamique, dans des compositions géométriques s'appuyant à la fois sur les lignes des instruments et sur des organisations circulaires autour d'une polarité centrale vers laquelle ces lignes convergent. Ces caractéristiques sont en particulier celles des groupes de jazz noirs de la Harlem Renaissance, souvent photographiés avec des effets d'ombres et des compositions d'images " chargées », principes que l'on retrouve aussi dans les tableaux de ce mouvement, par exemple ceux d'Aaron Douglas. Une photographie célèbre de Duke Ellington (figure 5) ${ }^{10}$ résume assez bien ces effets d'accumulation des musiciens, de création de rythmes visuels par les axes des instruments et de présence de musiciens sous formes d'ombres à l'arrière-plan.

9 On trouvera beaucoup de photographies de groupes de jazz des années 1910 et 1920, noirs et blancs, sur le site : http://www.polarityrecords.com/early-jazz-bands-and-jug-bands-photo-gallery.html, consulté le $1^{\text {er }}$ septembre 2017.

10 La page de Wikimedia Commons indique que la photographie provient de la première édition de l'ouvrage en 1936, mais il s'agit en fait de l'iconographie de la deuxième édition en 1943 (voir https://commons.wikimedia.org/wiki/File:Maud Cuney Hare-136-Duke Ellington Orchestra.jpg et les versions en ligne des deux éditions de l'ouvrage : http://digital.library.upenn.edu/women/cuney-hare/ musicians/musicians.html, consultés le $1^{\mathrm{er}}$ septembre 2017). 


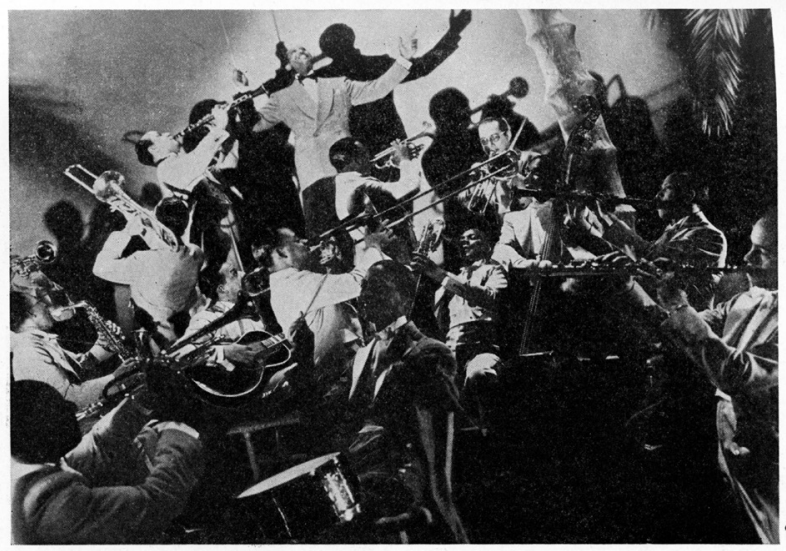

Figure 5 : Photographie de Duke Ellington et son orchestre, parue dans Cuney-Hare 1943, p. 136.

Le cinéma s'empare précisément de ces caractéristiques, indépendamment de la musique concernée. Si, proportionnellement aux autres numéros hollywoodiens, il y a dans les numéros à instruments plus de musiciens « non blancs » que dans l'ensemble du corpus, la domination des blancs reste évidemment écrasante en période de ségrégation ${ }^{11}$. Mais les musiciens noirs trouvent une place particulière dans ce type de numéros. Ils s'y trouvent très fréquemment filmés avec des effets rappelant le style des représentations de la Harlem Renaissance, par exemple Louis Armstrong dans le numéro fantastique "Skeletons in the Closet" de Pennies from Heaven (Norman Z. McLeod, 1936) (extrait vidéo 9), où le découpage montre un espace "impossible » puisque les positions relatives des musiciens et la lumière diégétique sont délibérément incohérents d'un plan à l'autre. Armstrong lui-même intervient alternativement en chair et en os et sous forme d'ombre presque surnaturelle (figure 6a). De même que les big bands blancs doivent une partie de leurs influences musicales aux groupes de jazz noirs, l'iconographie qui leur est associée dans les films, relève aussi de traditions visuelles associées à ces groupes, comme le montre par exemple le générique de Sun Valley Serenade, où le groupe de Glenn Miller apparaît sous forme d'ombres. Plus largement, les ombres des musiciens sont progressivement associées à des types de musiques extrêmement variés (figures $6 b$ et $6 c$ ).
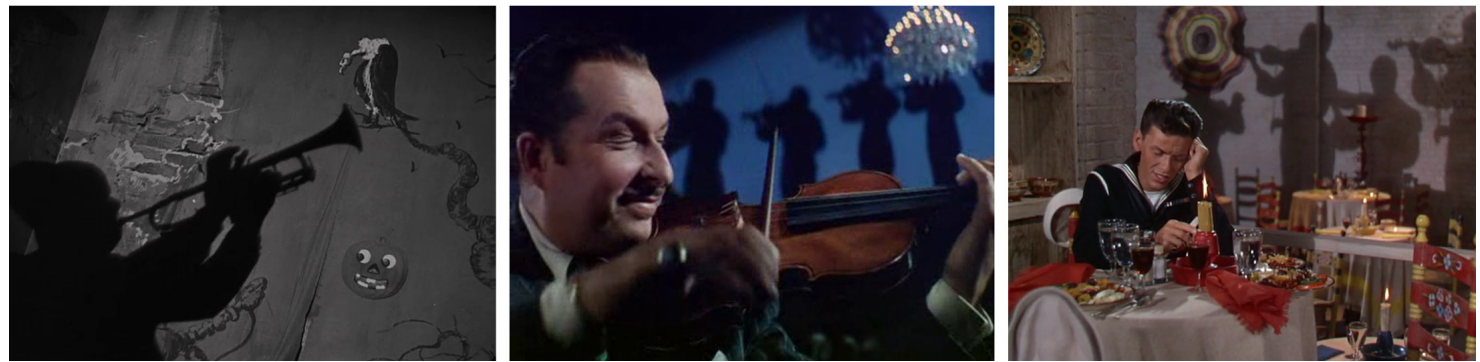

Figures 6a, 6b et 6c: Jeux d'ombres : a) Louis Armstrong dans "Skeletons in the Closet " (Norman Z. McLeod, Pennies from Heaven, 1936); b) Xavier Cugat dans "Alma Llanera " (George Sidney, Bathing Beauty, 1944); c) Frank Sinatra accompagné par les ombres des musiciens dans "What Makes the Sunset " (George Sidney, Anchors Aweigh, 1945).

11 Pour des précisions sur ces questions raciales dans le cas des musiques de jazz, voir Mouëllic 2000, p. $16-28$ 


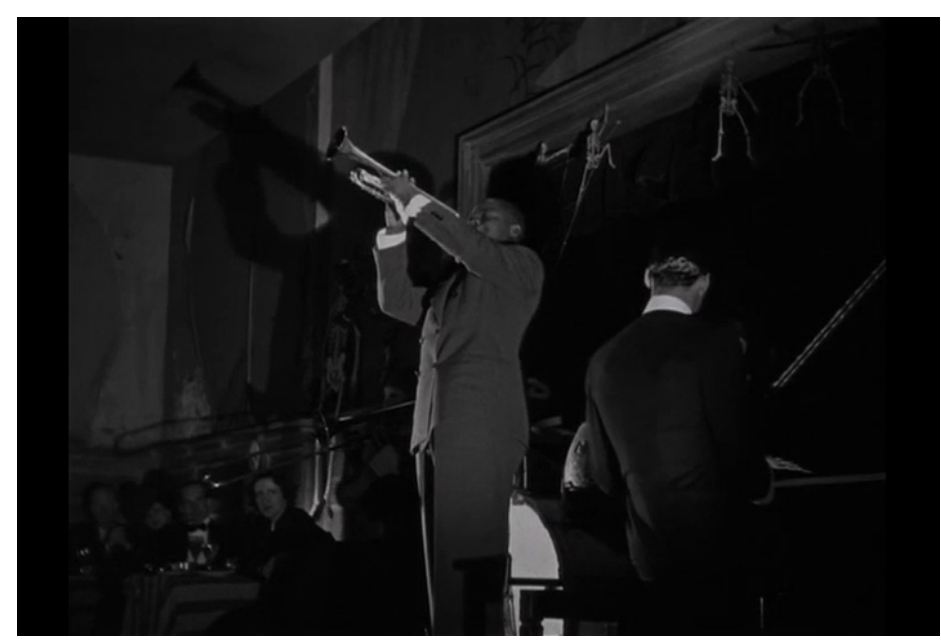

Extrait vidéo 9: Norman Z. McLeod, Pennies from Heaven (1935), "Skeletons in the Closet" par Louis Armstrong (C) Emanuel Cohen Productions/Columbia.

\section{L'orchestration visuelle}

La troisième grande caractéristique esthétique des numéros à instruments repose sur la synchronisation entre son et image et la manière de donner à voir la musique. D'une part on y trouve des mouvements de caméra parfois complexes, qui encerclent les instrumentistes et se superposent au rythme musical. Il peut s'agir d'un balayage général de la caméra qui apporte une intensité visuelle (parmi les exemples déjà évoqués, "Going Up » de Duke Ellington) ; ou de mouvements qui " ciblent » un par un les musiciens en passant de l'un à l'autre pour donner le sentiment de coïncidences entre la quête de la caméra et le surgissement d'un solo (le premier numéro de Tommy Dorsey dans Du Barry Was a Lady, "I'm Getting Sentimental Over You " en est un exemple particulièrement efficace, comme " Heat Wave » dans Alexander's Ragtime Band). D'autre part, l'autre grand modèle formel repose sur le montage dont la rythmique visuelle recouvre celle du morceau. Que le montage soit pratiqué « en direct » par le mouvement de la caméra, ou fait à la table ensuite, il s'agit toujours de chercher la coïncidence entre le défilement de l'image et l'écoute.

Le montage passant d'un pupitre à l'autre est particulièrement utilisé avec les musiques déjà orchestrées par alternance de phrases entre les différents instruments, misant sur des changements de timbres. Quand la musique possède ce type de " montage " propre, la réalisation l'illustre souvent littéralement, par exemple dans de nombreux numéros de Strike Up the Band. Dans «When Day Is Done » (extrait vidéo 10), le mixage sonore et l'image fonctionnent parfaitement ensemble : dans la transition entre trompette et saxophone, par exemple, le son est rééquilibré parallèlement à la focalisation de la caméra en inversant la hiérarchie au moment où l'image change d'instrumentiste. Le début du finale du même film adopte lui aussi ce type de découpage, avec des coupes plus nettes. Là encore, l'iconographie est indépendante du répertoire musical : la fugue de Bach dirigée par Leopold Stokowski dans The Big Broadcast of 1937 (Mitchell Leisen, 1936) est montée exactement de la même manière, l'image donnant à voir les différentes entrées d'instruments et rendant parfaitement lisible l'écriture musicale (extrait vidéo 11). 


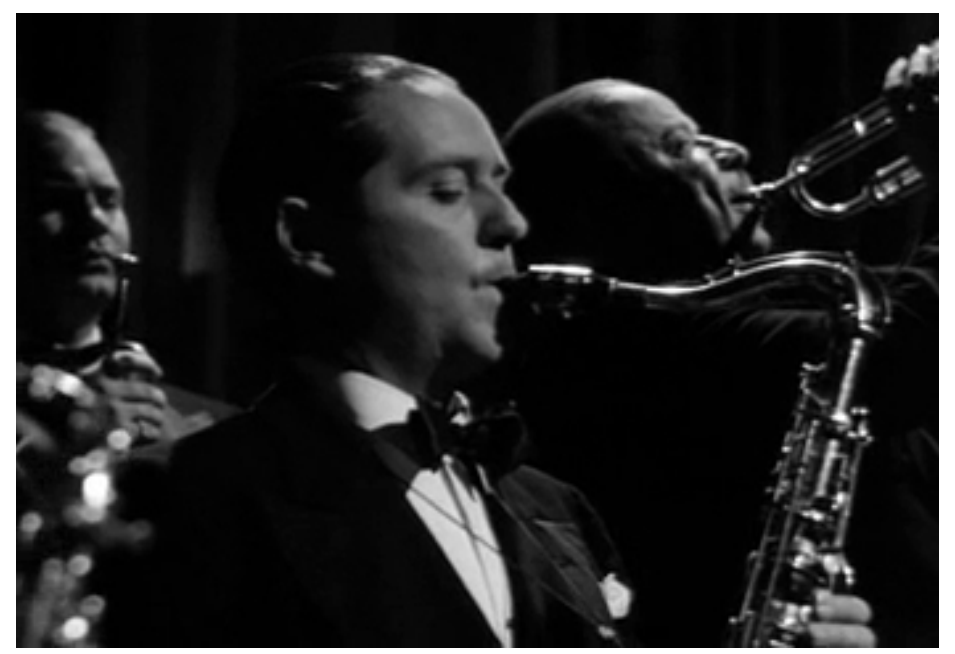

Extrait vidéo 10 : Busby Berkeley, Strike Up the Band (1940), "When Day Is Done " par Paul Whiteman et son orchestre (C) MGM.

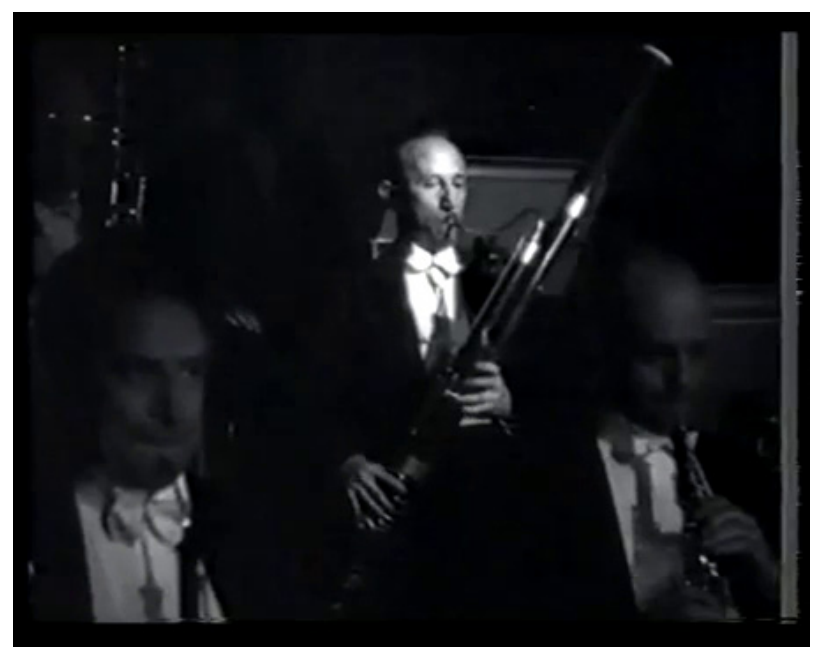

Extrait vidéo 11 : Mitchell Leisen, The Big Broadcast of 1937 (1936), version symphonique d'une fugue de Bach (C) Paramount.

Comme pour le montage, la composition des plans elle-même adopte un principe commun indépendant du style musical, souvent par "série », visant à faire ressortir la forme des instruments plutôt que les visages individuels des instrumentistes (figures 7a, $7 \mathrm{~b}$ et $7 \mathrm{c}$ ). Il y a certes des variantes (les trombones de l'orchestre de Philadelphie sont moins inclinés que ceux de Glenn Miller), mais l'effet recherché est voisin : il s'agit de faire ressortir l'harmonie précisément et la synchronisation des musiciens entre eux, qui fait partie des aspects les plus visibles de la musique, et que l'on voit rarement aussi bien sur une scène. Les numéros les plus travaillés formellement sont ainsi ceux qui abolissent l'espace, alors que la spatialisation et la position relative des instruments sont très importantes pour la musique scénique. Le cinéma préfère superposer des détails du travail d'ensemble, la coordination des gestes, dans des formes qui perdent parfois toute notion de scénographie. 

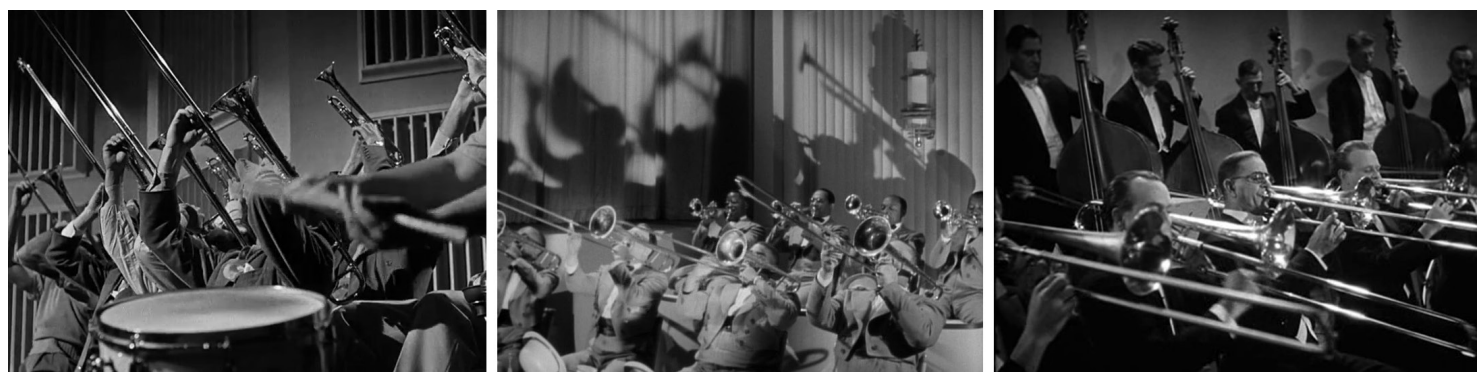

Figures 7a, 7b et 7c: Variations sur les lignes de trombones : a) l'orchestre de Glenn Miller dans "People Like You and Me» (Archie Mayo, Orchestra Wives, 1942) ; b) l'orchestre de Cab Calloway dans "Jumpin' Jive" (Andrew L. Stone, Stormy Weather, 1943); c) l'orchestre philharmonique de Philadelphie dirigé par Leopold Stokowski (Henry Koster, One Hundred Men and a Girl, 1937).

\section{SINGULARITÉS DES MUSICIENS}

Une manière de filmer aussi homogène pourrait estomper la singularité des musiciens, a fortiori parce que prise de son et prise de vue sont réalisées séparément, et que le numéro musical n'est jamais la trace d'une performance unique mais une recomposition, en cela plus proche du disque que des médias de diffusion live. Si l'on regarde de près, ces numéros comportent de surcroît beaucoup de plans «trichés " ou de faux raccords : les positions relatives des instruments bougent souvent au fil du montage. La clarinette basse posée à côté des saxophonistes au début de « The Jumpin' Jive » (Stormy Weather) disparaît ainsi en cours de séquence (extrait vidéo 12), avant que l'espace de la scène subisse lui-même une transformation analogue. À la fin de "People Like You and Me »(Orchestra Wives), une caisse claire apparaît fugitivement devant les trombones (figure 7a) pour introduire un nouvel effet de cadrage (au moment d'une citation de la marche militaire « Yankee Doodle Dandy »).

Extrait vidéo 12 : Andrew L. Stone, Stormy Weather (1935), " Jumpin' Jive » par Cab Calloway. Extrait remonté (C) Twentieth Century-Fox.

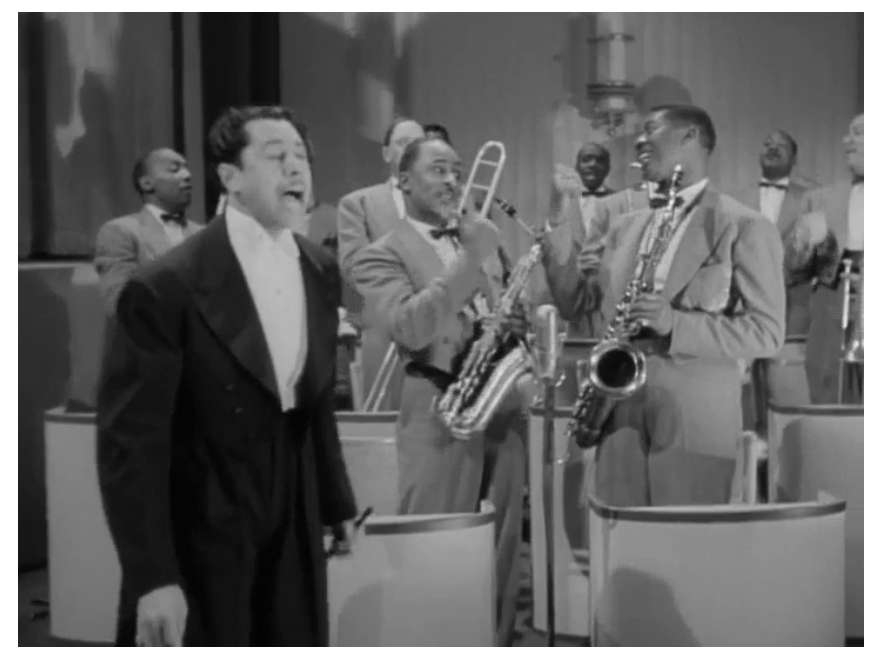

Non seulement les morceaux filmés sont l'objet d'une reconstruction, mais le cinéma a aussi des conséquences sur la pratique des musiciens. Habitués à s'adapter à différents espaces selon les lieux où ils se produisent, les groupes sont particulièrement amenés à ajuster leur placement dans les films. Généralement, un groupe a ses 
repères, un principe de disposition propre. Les archives du régisseur du Paramount Theatre montrent que les artistes demandaient sur scène une organisation du plateau minutieuse. Chaque ensemble pouvait placer assez différemment les instruments, même les plus encombrants comme le piano : ce n'est pas le groupe qui s'adapte entièrement à la salle, mais la salle qui essaie de reproduire l'organisation demandée par les musiciens.

La comparaison des dispositions des groupes en live avec celle des films est tout à fait passionnante car elle montre la capacité " transformante " de Hollywood. Pour donner des conclusions définitives, il faudrait consacrer un article entier à chacun des musiciens et s'appuyer aussi sur d'autres sources, des corpus de photographies, et d'autres formes audiovisuelles comme les courts-métrages musicaux de nature plus " documentaire », par exemple les concerts filmés dans certains reportages d'actualités. Je voudrais seulement ici faire quelques observations pour amorcer ce type de recherche.

Les dispositions de groupes retenues dans les films hollywoodiens s'expliquent par un faisceau d'éléments : la souplesse de l'interprète lui-même, son image et en particulier ses caractéristiques ethniques et raciales, et le parti-pris du studio. Parmi les musiciens noirs, Louis Armstrong semble particulièrement fluide : le trompettiste lui-même a une carrière extrêmement diversifiée et une capacité à jouer dans des formations variables. Ses interventions dans High Society (Charles Walters, 1956) correspondent à ce dont témoignent les photographies et les informations sur un concert donné au Paramount Theatre ${ }^{12}$ : il y a autant de dispositions que de morceaux. Seul son deuxième numéro du film reproduit un placement adopté au Paramount Theatre avec le piano à gauche et la contrebasse derrière. Il semblerait que la polyvalence d'Armstrong rende indifférente la place des musiciens qui l'accompagnent. En revanche, Duke Ellington est quant à lui extrêmement constant dans le positionnement de ses musiciens sur scène, du moins au Paramount Theatre. Il place systématiquement le piano au milieu (un Steinway qu'il exige pour l'occasion), éloigne le plus possible les percussions qui sont à côté de lui, et ses musiciens jouent avec un pupitre. Au contraire, il apparaît dans Cabin in the Sky (extrait vidéo 5) avec une image beaucoup plus détendue : pas de pupitres pour donner un sentiment d'improvisation, disposition confuse et modeste piano droit ouvert. Une étude plus précise des archives de tournage nous en apprendrait davantage sur les responsabilités du musicien et des producteurs dans cette décision. On peut toutefois formuler l'hypothèse selon laquelle Hollywood s'intéresse aux musiciens noirs pour la décontraction et l'énergie qu'ils représentent, ce qui demande d'effacer les signes d'une forme de rigueur et de décorum.

Du côté des groupes latins, très en vogue au début des années 1940, Xavier Cugat est certainement la vedette principale. Les cinq configurations de concerts donnés au Paramount Theatre entre 1940 et 1953 sont des variations sur un principe commun : les bois et cuivres à droite, les deux tambours cubains de part et d'autre au 
premier rang, le marimba devant sur le côté. Cette base est déclinée dans la plupart de ses numéros cinématographiques, mais avec des variantes qui semblent liées au studio : "Chiu Chiu » (extrait vidéo 13), dans You Were Never Lovelier (William A. Seiter, 1942) semble le plus proche de ce que Cugat faisait en live, à la différence que l'on voit plus de violons à l'écran pour créer des effets de série (quatre ou cinq, au lieu des deux habituels sur scène) et que le marimba, qui est mis en valeur au théâtre par sa place à l'avant-scène, est quasiment invisible à l'écran dans les plans d'ensemble et plutôt mis en valeur par des gros plans sur le clavier. Les autres films avec Cugat s'éloignent de plus en plus de ce modèle : les percussions cubaines se multiplient et sont disposées aussi à l'arrière-plan pour créer de la profondeur et renforcer la touche exotique (dans son numéro de Stage Door Canteen par exemple). Il place ses choristes sur les côtés alors que sur scène ils sont devant. Bref, il semblerait que Hollywood privilégie les instruments exotiques en tant qu'objets et les rende visibles par tous les moyens possibles. Les films de sa période MGM sont l'occasion de recherches iconographiques et d'une forme de déconstruction des pratiques scéniques du groupe : démultiplication du nombre d'instrumentistes, effectif plus important, moins de plans d'ensemble et plus de détails pour abolir l'espace et laisser la place à des explorations plastiques, notamment dans l'usage de la couleur, par exemple dans "Alma Llanera » où la disposition des musiciens est en réalité impossible (extrait vidéo 14). À la fin des années 1940 à la MGM, Cugat et ses musiciens portent des tenues parfois moins clinquantes et moins exotiques dans des numéros qui défont le groupe en le transformant en une sorte de réseau abstrait (comme « Jungle Rhumba » dans Neptune's Daughter (Edward Buzzell, 1949).

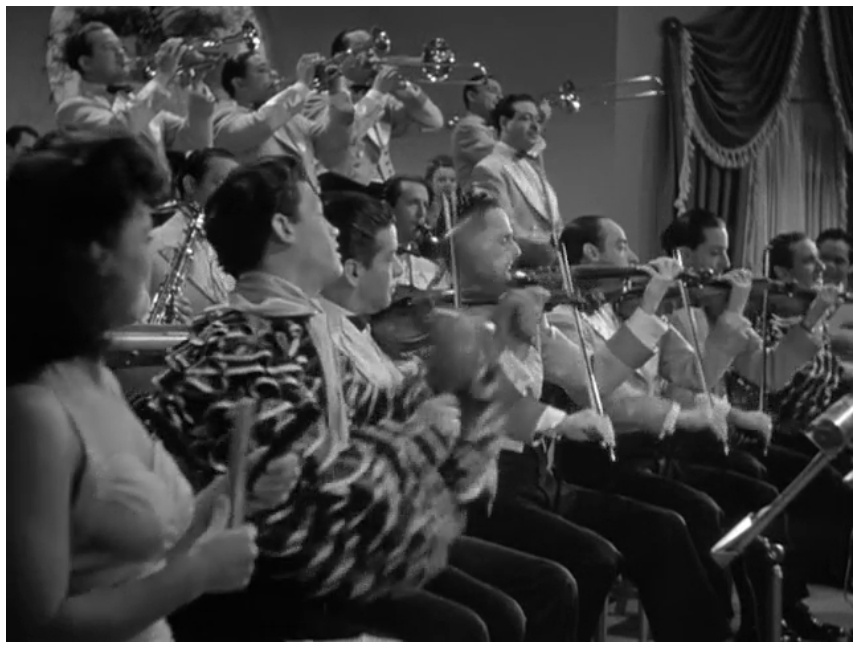

Extrait vidéo 13 : William A. Seiter, You Were Never Lovelier (1942), "Chiu Chiu " par Xavier Cugat (C) Columbia. 
Extrait vidéo 14: George Sidney, Bathing Beauty (1944), "Alma Llanera " par Xavier Cugat (C) MGM.

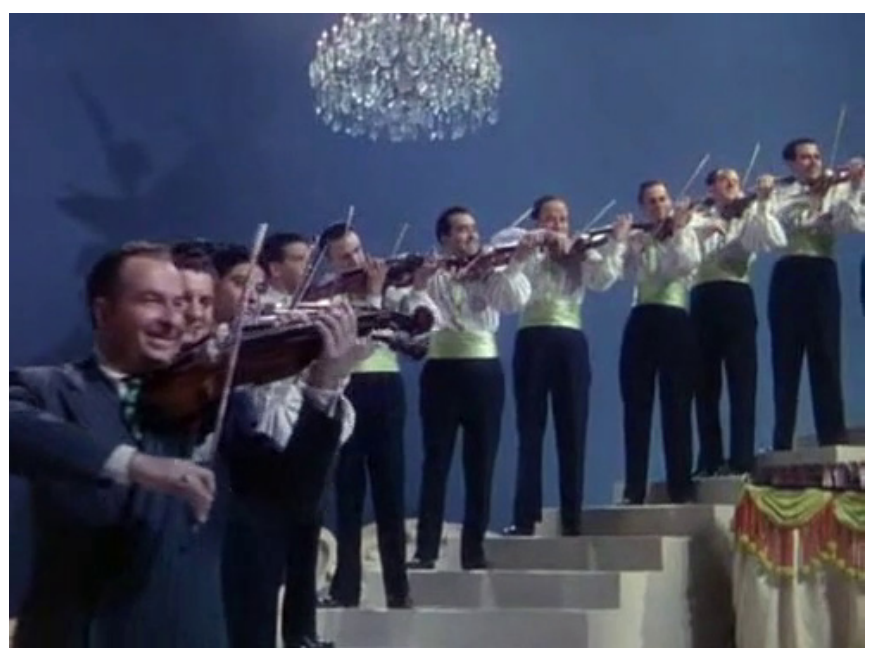

Du côté des big bands blancs, on peut noter que plus ils tournent pour Hollywood, plus la nécessité de diversifier les numéros se fait sentir, avec celle de préserver leur organisation " originelle " dans certains. Les films conservent très souvent les séries d'estrades utilisées par ces groupes sur scène, notamment pour surélever le batteur au fond. L'orchestre de Glenn Miller est disposé de façon analogue à ce qu'il fait au Paramount Theatre dans certains cas, comme "In the Mood " dans Sun Valley Serenade en 1941, qui est son grand succès de l'année précédente. Mais le nombre de numéros assurés impose une nécessité de variation sur le plan visuel, a fortiori dans le film suivant, Orchestra Wives où l'orchestre adopte des dispositions de plus en plus fluctuantes. Quant à Benny Goodman, il fait preuve d'une certaine souplesse sur scène, ce qui a sans doute facilité la conception de numéros cinématographiques. "Paducah » dans The Gang's All Here (Busby Berkeley, 1943) donne une allure de spirale à une disposition proche de celles utilisées au Paramount Theatre, et tire ses effets de l'ornementation apportée par la réalisation avec ses couleurs changeantes et de mouvement de caméra qui ne révèle la scénographie globale qu'à la fin. L'ouverture très invraisemblable de Hollywood Hotel (Berkeley, 1938), qui montre les musiciens en parade, placés individuellement sur des capots de voitures qui roulent, permet à la suite du film - par exemple la reprise du célèbre «Sing, Sing, Sing » (extrait vidéo 15) - de revenir à des placements que le groupe a utilisés en live. Dans ces exemples chez Miller et Goodman, les chansons déjà connues sont filmées avec un placement du groupe proche de ses habitudes scéniques, et les variations sont introduites pour les morceaux originaux du film. 


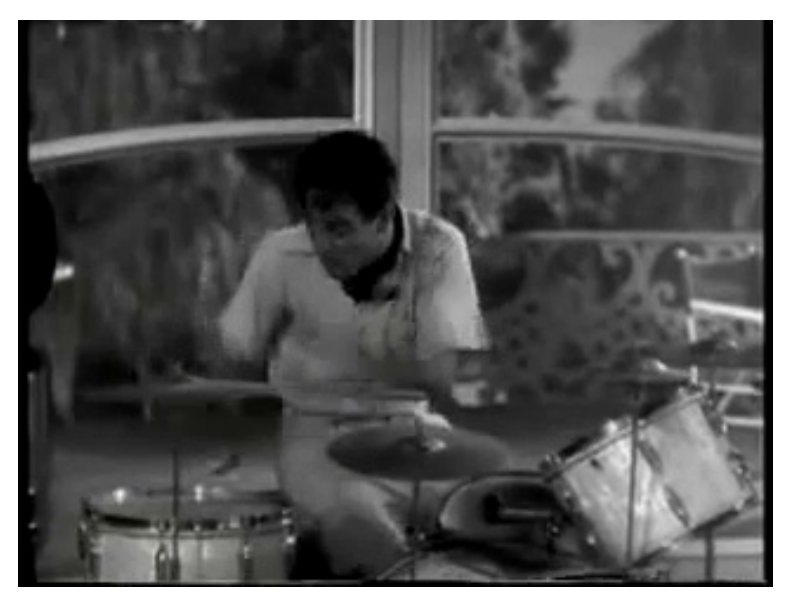

Extrait vidéo 15 : Busby Berkeley, Hollywood Hotel (1938), «Sing, Sing, Sing» (C) Warner Bros.

\section{Culture savante ET MusiQues POPUlaires : un ESPACE DE RÉCONCILIATION?}

Si ces détails appellent autant de recherches spécifiques sur les artistes pour les comprendre plus finement, ce qui les réunit est le traitement comparable que leur réserve Hollywood qui y voit des attractions de même nature, qu'il s'agisse des bigs bands, de Cugat et des groupes exotiques ou de musique classique. La relative unité esthétique a ainsi des effets culturels. Jane Feuer a montré à quel point le musical cinématographique exprime une méfiance vis-à-vis des formes trop élitistes et s'est fait une spécialité de confronter cultures savante et populaire pour se moquer de la première (Feuer 1993, p. 54 et suivantes). Plusieurs numéros à instruments illustrent bien ce principe, comme les sabotages des Marx Brothers dans A Night at the Opera (Sam Wood, 1935) ou Gracie Allen dans un " Concerto pour index » de Two Girls and a Sailor (Richard Thorpe, 1944 (extrait vidéo 16)). Mais une grande partie est le lieu d'une négociation plus complexe entre la culture de masse et la culture savante.

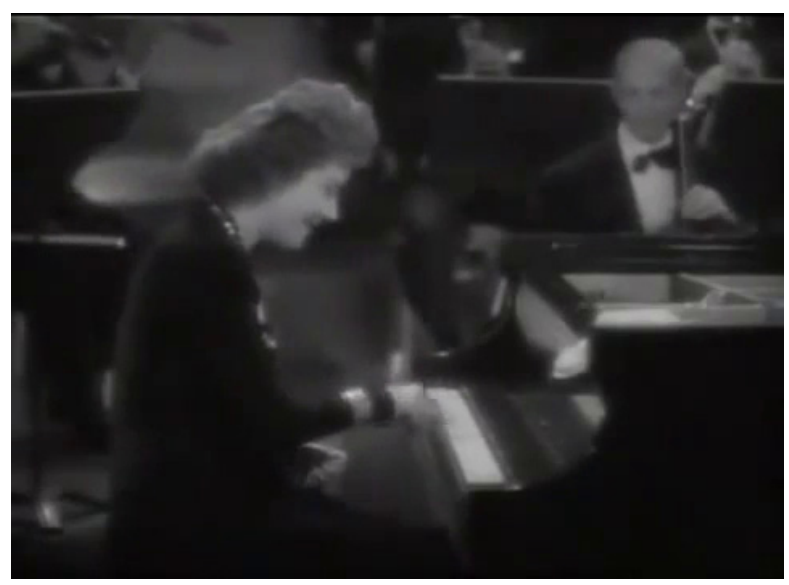

Extrait vidéo 16: Richard Thorpe,

Two Girls and a Sailor (1944), "Concerto pour index" avec Gracie Allen (C) MGM.

Cette confrontation est mise en jeu par les numéros à instruments et l'un des arguments de promotion pour The Big Broadcast of 1937 présente ainsi le film comme le duel au sommet du classique (Stokowski) et du swing (Goodman) (figure 8). Mais, d'une part, le duel ne se conclut pas nécessairement par l'échec des formes savantes ; 
d'autre part, l'explicitation de la confrontation est moins importante que la mise en scène d'une forme d'œcuménisme musical et de figures traitant symboliquement les différents groupes comme des équivalents. Dans Two Girls and a Sailor, un plateau tournant substitue ainsi le groupe de Cugat à celui, plus classique et "blanc » de Harry James, en les présentant en parallèle sans valoriser l'un plutôt que l'autre. Il se joue dans les numéros à instruments un double mouvement de légitimation de certaines musiques contemporaines, par leur inscription dans les repères de la musique classique, et de revalorisation de cette musique classique, traitée ici de façon moins satirique que ce que Feuer souligne à propos du genre musical dans sa globalité.

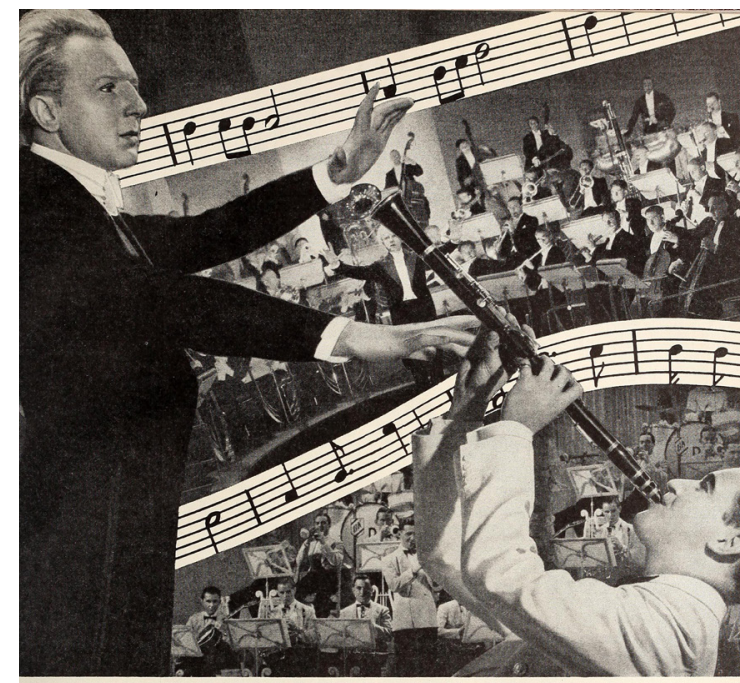

Figure 8: Publicité pour The Big Broadcast of 1937 parue dans Motion Picture Daily (29 septembre 1936, p. 3). Source: http://mediahistoryproject.org/.

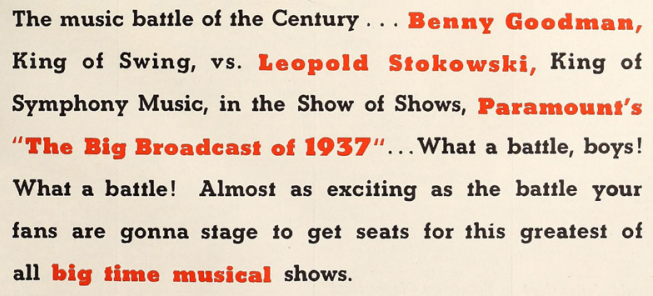

One of a Dozen Reasons "The Big Broadcast" Will Pay Off Plenty!

La légitimation du jazz est un enjeu qui dépasse Hollywood. Le célèbre concert de Benny Goodman à Carnegie Hall en janvier 1938 incarne cette reconnaissance et des films sortis peu après font écho à cet événement. C'est le cas d'Alexander's Ragtime $B a n d$, qui se termine lui aussi à Carnegie Hall, sommet de l'ascension de l'orchestre du protagoniste qui ressemble pour les derniers numéros (en particulier «Heat Wave »), à l'hybridation d'un orchestre symphonique et d'un big band. Ce fantasme d'occupation des grands auditoriums symphoniques s'exprime particulièrement ensuite dans Strike Up the Band, où Mickey Rooney rêve des grandes salles de Chicago où il finit par diriger triomphalement un orchestre qui semble d'abord symphonique, avant d'être agrémenté, par le miracle d'un medley, d'une section latine et d'une fanfare militaire dans un syncrétisme musical complet. 
Symétriquement, malgréla tendance pourla musique populaire à englober les formes classiques, le discrédit est loin d'être systématique dans les numéros à instruments fondés sur la musique savante. Dans Un Américain à Paris (Vincente Minnelli, 1951), le concerto pour piano de George Gershwin occupe une position stratégique à environ une heure du début du film - c'est par exemple la position de "Singin' in the Rain " dans le film du même titre, et celle de nombreux numéros à instruments. Dans Best Foot Forward, le rejet ponctuel de Beethoven pour du boogie woogie ne remet pas en question la nature hyper spectaculaire du morceau classique qui a précédé. Harry James incarne ailleurs 1'harmonie entre les styles, jouant aussi bien des concertos pour trompette de facture classique que du swing destiné aux danses de couples. Et la suite du film résiste à l'absorption du classique par la culture de masse : entraîné dans une danse de couple, Harry James s'y révèle maladroit et propose alors un numéro comique, aux antipodes des prestations virtuoses qu'il livre ailleurs. Le film montre l'échec du changement de registre du musicien, suggérant moins une assimilation qu'une coexistence du savant et du populaire.

Anchors Aweigh (George Sidney, 1945) est particulièrement intéressant, à la manière de plusieurs films de guerre comme Stage Door Canteen ou Thousands Cheers (George Sidney, 1943) qui postulent une sorte d'indifférence des catégories par la structure de la revue juxtaposant des numéros hétéroclites. Le numéro latin "Jealousy » (extrait vidéo 17), chanté par la soprano Kathryn Grayson, accorde une place croissante aux instrumentistes, alors que le film pourrait tout à fait se contenter d'échanges de regards romantiques entre elle et Gene Kelly qui la contemple béatement. La dimension proprement spectaculaire dépasse l'enjeu narratif et aboutit à l'interprétation de vocalises lyriques au milieu de marimbas et claves. Plus loin dans le film, José Iturbi insère un blues dans un morceau de facture classique, "The Donkey Serenade », comme une citation et non une substitution, ce qui conduit à une conclusion où il apparaît moins comme un concertiste classique qu'un chef de big band dirigeant un ensemble symphonique.

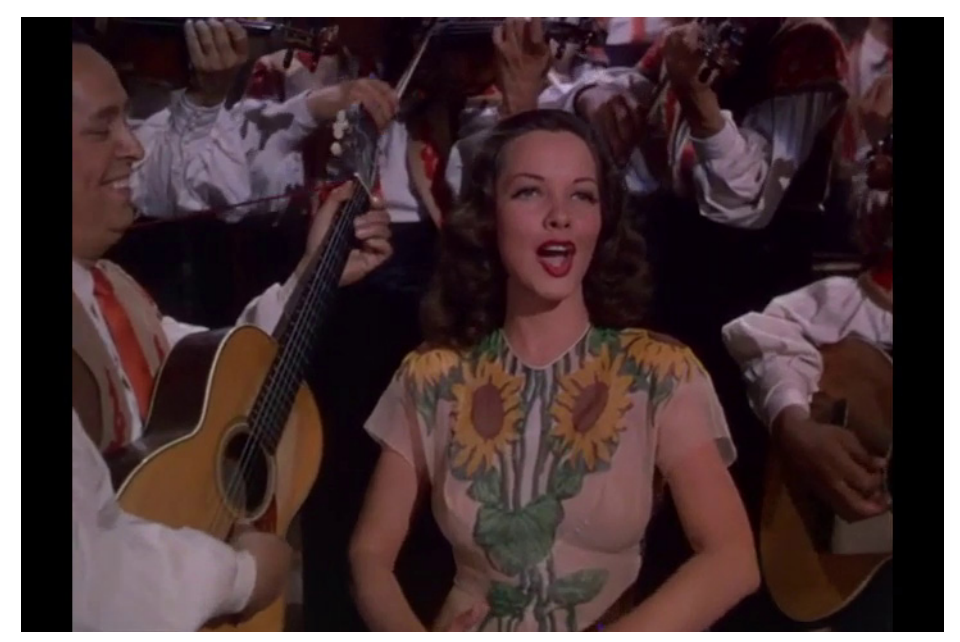

Extrait vidéo 17: George Sidney, Anchors Aweigh (1945), «Jealousy» par Kathryn Grayson (C) MGM.

Plusieurs figures du monde de la musique classique ont ainsi connu leur heure de gloire à Hollywood, comme le chef Leopold Stokowski qui, quelques années 
avant Bernstein, s'était donné le même projet de rendre son répertoire accessible. Dans son film avec Deanna Durbin, One Hundred Men and a Girl (Henry Koster, 1937), le premier numéro correspond aux stéréotypes pointés par Feuer : on voit des plans de réaction sur le public associant un spectateur passionné et un autre manifestement ennuyé, endormi... Mais la fin du numéro (extrait vidéo 18) renverse cette situation en renonçant aux plans de réaction et en mettant en valeur les séries d'instruments dans une sorte de montage d'attractions conclusif qui revalorise ainsi le contenu musical. Le film entier est une comédie musicale reposant presque exclusivement sur le répertoire classique et Stokowski est traité comme une star : il bénéficie de plans isolés sur ses mains, présentées comme celles d'un instrumentiste, et surtout celles qui contrôlent la musique, la mise en scène suggérant la magie et la puissance qu'il y a à diriger une force aussi vaste que celle d'un orchestre symphonique.

Extrait vidéo 18: Henry Koster,

One Hundred Men and a Girl (1937),

Fin du premier numéro dirigé par

Leopold Stokowski C) Universal.

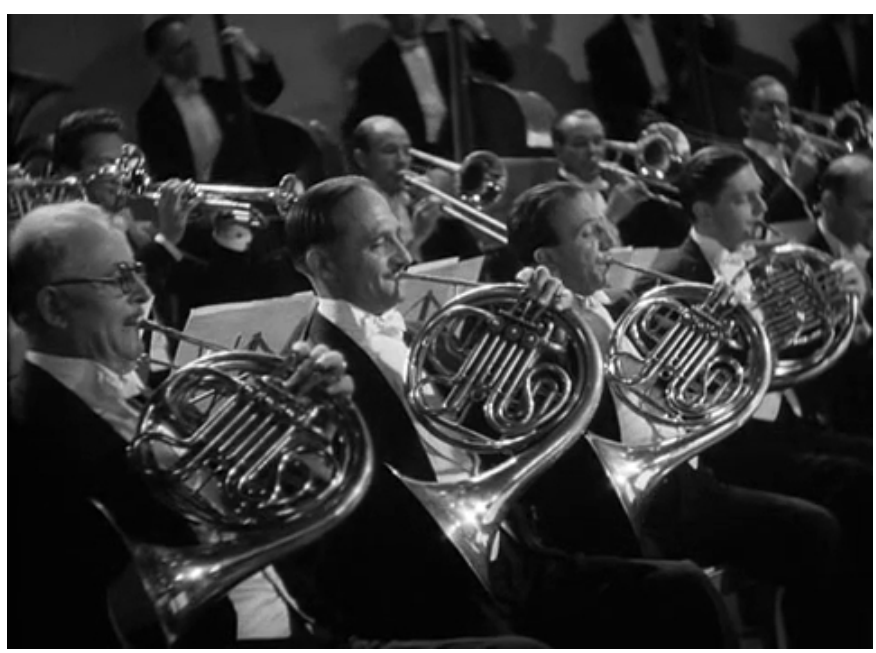

\section{CONCLUSION}

Si les numéros à instruments peuvent se décliner selon des modalités diverses dans le film musical hollywoodien, reflétant à eux seuls le foisonnement et la variété du genre, ils n'en constituent pas moins un archétype spectaculaire important. Au-delà des singularités, celui-ci se définit d'abord par son contenu (montrer l'acte de jouer d'un instrument), mais aussi par les trois aspects que j'ai décrits ici : il s'agit d'abord pour Hollywood de se démarquer du musical théâtral et de la musique radiophonique, en mettant en avant ses formes musicales propres. Deuxièmement, les numéros présentent pour une grande partie des traits stylistiques liés à la rhétorique de l'attraction, une suspension du récit au profit d'une esthétique de la sensation, notamment pour faire ressentir les rythmes, les timbres ${ }^{13}$... Certains de ces traits sont aussi la réappropriation et la transformation par l'industrie du cinéma d'éléments empruntés à des musiques spécifiques (notamment les musiques noires) et appliqués

13 Pour un approfondissement sur la définition de l'attraction, voir Paci 2011, notamment le chapitre 3. 
à d'autres répertoires. Troisièmement, les numéros à instruments sont le lieu principal du syncrétisme musical hollywoodien et d'un nivellement des catégories culturelles : il y est plus question de la musique dans son principe général, que de particularités stylistiques, par exemple.

Il s'agissait ici de tracer les grandes lignes de l'analyse et de proposer des perspectives de recherches futures : d'une part en défendant le principe de poursuivre l'étude du film musical en travaillant à échelle du numéro, ce qui impliquera d'entrer dans des analyses plus détaillées de chacun; d'autre part en mettant en relief une catégorie particulière de numéros encore peu étudiée. Ainsi, les numéros à instruments, qui pourraient paraître plus faibles en contenus que les grands ballets à la Un Américain à Paris, n'en sont pas moins constitutifs du genre musical et révèlent l'intérêt que Hollywood a toujours cultivé pour la musique indépendamment de la danse. Ils présentent aussi des enjeux idéologiquement assez complexes, l'abstraction dominant sur le plan esthétique et les catégories culturelles y étant moins fortement définies.

La mise en avant de tels archétypes spectaculaires révèle bien la nécessité de poursuivre leur histoire intermédiale, et de retracer le parcours de formes courtes, indépendamment de la narration et de l'unité des films : les numéros de diffusion médiatisée de la musique (broadcasting), les types de chant (comme la berceuse, la déploration) ou les rites de musique collective (les numéros gospel ou à valeur religieuse) sont autant d'autres exemples qui mériteraient une histoire propre.

\section{FILMOGRAPHIE}

Anderson, John Murray (1930), King of Jazz, Universal.

Berkeley, Busby (1935), Gold Diggers of 1935, Warner Bros.

Berkeley, Busby (1938), Hollywood Hotel, Warner Bros.

Berkeley, Busby (1940), Strike Up the Band, MGM.

Borzage, Frank (1943), Stage Door Canteen, Sol Lesser Productions.

Cukor, George (1954), A Star Is Born, Warner Bros.

Del Ruth, Roy (1943), Du Barry Was a Lady, MGM.

Koster, Henry (1937), One Hundred Men and a Girl, Universal.

Leisen, Mitchell (1936), The Big Broadcast of 1937, Paramount.

Mayo, Archie (1942), Orchestra Wives, Twentieth Century-Fox.

McLeod, Norman Z. (1935), Pennies from Heaven, Emanuel Cohen Productions/Columbia.

Minnelli, Vincente (1943), Cabin in the Sky, MGM.

Seiter, William A. (1942), You Were Never Lovelier, Columbia.

Sidney, George (1944), Bathing Beauty, MGM.

Sidney, George (1945), Anchors Aweigh, MGM.

Sidney, George (1945), Kiss Me, Kate, MGM.

Stone, Andrew L.(1935), Stormy Weather, Twentieth Century-Fox.

Thorpe, Richard (1944), Two Girls and a Sailor, MGM. 


\section{BIBLIOGRAPHIE}

Altman, Rick (1987), The American Film Musical, Bloomington \& Indianapolis, Indiana University Press ; La comédie musicale hollywoodienne. Les problèmes de genre au cinema, traduit de l'anglais par Jacques Lévy, Paris, Armand Colin, 1992.

Altman, Rick (1999), " De l'intermédialité au multimédia. Cinéma, médias, avènement du son ", CiNéMAS. Revue d'études cinématographiques, vol. 10, nº 1, 1999, p. 37-53.

Chapman, James (2006), " A Short History of the Big Band Musical ", dans Ian Conrich et Estella Tincknell (dir.), Film's Musical Moments, Edinburgh, Edinburgh University Press, p. 28-41.

Cuney-Hare, Maud (1943), Negro Musicians and Their Music [1936], Washington, Associated Publishers.

Feuer, Jane (1993), The Hollywood Musical, Bloomington, Indiana University Press.

Gabbard, Krin (2003), " Biographies de jazzmen. Hollywood clichés », traduit de l'anglais par Baptiste Piégay, dans Franco La Polla (dir.), All That Jazz. Un siècle d'accords et désaccords avec le cinéma, Paris, Cahiers du cinéma, p. 65-86.

Masson, Alain (1981), Comédie musicale, Paris, Stock.

Mouëllic, Gilles (2000), Jazz et cinéma, Paris, Cahiers du Cinéma.

Paci, Viva (2011), La comédie musicale et la double vie du cinéma, Lyon/Udine, Aléas/Forum.

Rubin, Martin (1993), Showstoppers. Busby Berkeley and the Tradition of Spectacle, New York, Columbia University Press. 